\title{
The impact of fluid intervention on complications and length of hospital stay after pancreaticoduodenectomy (Whipple's procedure)
}

\author{
Laurence Weinberg $^{1 *}$, Derrick Wong ${ }^{2}$, Dharshi Karalapillai ${ }^{3}$, Brett Pearce ${ }^{2}$, Chong O Tan ${ }^{4}$, Stanley Tay ${ }^{5}$,
} Chris Christophi ${ }^{6}$, Larry McNicol ${ }^{1}$ and Mehrdad Nikfarjam ${ }^{7}$

\begin{abstract}
Background: There is limited information on the impact on perioperative fluid intervention on complications and length of hospital stay following pancreaticoduodenectomy. Therefore, we conducted a detailed analysis of fluid intervention in patients undergoing pancreaticoduodenectomy at a university teaching hospital to test the hypothesis that a restrictive intravenous fluid regime and/or a neutral or negative cumulative fluid balance, would impact on perioperative complications and length of hospital stay.

Methods: We retrospectively obtained demographic, operative details, detailed fluid prescription, complications and outcomes data for 150 consecutive patients undergoing pancreaticoduodenectomy in a university teaching hospital. Prognostic predictors for length of hospital stay and complications were determined.

Results: One hundred and fifty consecutive patients undergoing pancreaticoduodenectomy were evaluated between 2006 and 2012. The majority of patients were, middle-aged, overweight and ASA class III. Postoperative complications were frequent and occurred in 86 patients (57\%). The majority of complications were graded as Clavien-Dindo Class 2 and 3. Postoperative pancreatic fistula occurred in 13 patients (9\%), and delayed gastric emptying occurred in 25 patients (17\%). Other postoperative surgical complications included sepsis (22\%), bile leak (4\%), and postoperative bleeding (2\%). Serious medical complications included pulmonary edema (6\%), myocardial infarction (8\%), cardiac arrhythmias (13\%), respiratory failure (8\%), and renal failure (7\%). Patients with complications received a higher median volume of intravenous therapy and had higher cumulative positive fluid balances. Postoperative length of stay was significantly longer in patients with complications (median 25 days vs. 10 days; $\mathrm{p}<0.001$ ). After adjustment for covariates, a fluid balance of less than 1 litre on postoperative day 1 and surgeon caseloads were associated with the development of complications.
\end{abstract}

Conclusions: In the context of pancreaticoduodenectomy, restrictive perioperative fluid intervention and negative cumulative fluid balance were associated with fewer complications and shorter length of hospital stay. These findings provide good opportunities to evaluate strategies aimed at improving perioperative care.

\section{Background}

It is recognized that liberal fluid administration is common practice after major hepatobiliary and pancreatic surgery [1]. However, there is limited information on perioperative fluid therapy and its impact on complications and length of hospital stay following pancreaticoduodenectomy (PD). Whilst enhanced recovery surgical

\footnotetext{
* Correspondence: laurence.weinberg@austin.org.au

'Department of Anesthesia, Department of Surgery, University of Melbourne, Austin Hospital, Heidelberg, Australia

Full list of author information is available at the end of the article
}

programs may reduce the length of hospital stay following PD $[2,3]$, the independent role of intravenous fluid therapy remains unclear due to the complex nature of such programs and the lack of randomized controlled trials. Therefore, we conducted a retrospective detailed analysis of detailed fluid intervention in patients undergoing PD at a university teaching hospital to determine the impact of fluid therapy on complications and length of hospital stay. We tested the hypothesis that a restrictive intravenous fluid regime and/or a neutral or negative cumulative fluid balance would be associated with fewer

\section{Biomed Central}


perioperative complications and a reduced length of hospital stay.

\section{Methods}

After Austin Health Human Research Ethics Committee approval, we conducted a retrospective analysis of consecutive patients undergoing open PD at a university teaching hospital with expertise in hepatobiliarypancreatic surgery including liver transplantation. A total of 150 consecutive patients between January 2006 and November 2012 were included. Patients undergoing total, distal or completion pancreatectomy were excluded from analysis. Data was extracted from a prospectively managed electronic hospital database. Comprehensive cross checks using computerized medical records were reviewed by three independent investigators who checked that a thorough and accurate tally of fluid administration, losses and complications were recorded. All biochemical, hematologiocal, laboratory and radiological results were reviewed. Any complication coded by the hospital in the health information database was cross checked with clinical records to ensure that the complication was correctly reported and coded. Complications were recorded as unexpected events occurring during surgery or the postoperative period, with pancreatic leak and delayed gastric emptying graded and classified according to the International Study Group of Pancreatic Surgery [4-6]. Complications were graded according to Clavien-Dindo Classification [7]. Common Terminology Criteria for Adverse Events were classified according to the US Department of Health and Human Services, National Institute of Health and National Cancer Institute [8] and detailed in Table 1. Characteristics of the cohort recorded included patient demographics, body mass index, American Society of Anesthesiologists (ASA) class, comorbidities and preoperative laboratory tests. Operative details collected included pathology, anesthetic technique, the volume of cases performed by each surgeon, and intraoperative fluid administration. Specifics of intravenous fluid administration from the first to third postoperative days were collected including fluid type (crystalloid, colloid, blood) and daily fluid balances. Finally, data regarding clinical complications were compiled in conjunction with length of hospital stay.

We defined a restrictive fluid regime as: intraoperative fluid therapy $\leq 4$ litres, day 1 fluids $\leq 3$ litres, day 2 fluids $\leq 2$ litres, and day 3 fluids $\leq 1.5$ litres or a neutral or negative cumulative fluid balance at the same time points. A liberal fluid regime was defined as intraoperative fluid therapy $>5$ litres, day 1 fluids $>4$ litres, day 2 fluids $>3$ litres, and day 3 fluids $>2$ litres or a positive cumulative fluid balance at the same time points. This definition was selected to be consistent with the "REstrictive Versus LIbEral Fluid Therapy in Major Abdominal
Table 1 Summary of number of patients undergoing pancreaticoduodenectomy with complications

\begin{tabular}{|c|c|}
\hline Total patients undergoing pancreaticoduodenectomy & 150 \\
\hline Patients with complications & $86(57 \%)$ \\
\hline Clavien classification i & $5(3 \%)$ \\
\hline ii & $41(27 \%)$ \\
\hline iii & $25(17 \%)$ \\
\hline iv & $11(7 \%)$ \\
\hline$v$ & $3(2 \%)$ \\
\hline Post operative pancreatic fistula ${ }^{a}$ & $13(9 \%)$ \\
\hline Grade A & - \\
\hline Grade B & $4(3 \%)$ \\
\hline Grade C & $9(6 \%)$ \\
\hline Bile leak ${ }^{b}$ & $6(4 \%)$ \\
\hline Acute pancreatitis ${ }^{c}$ & $8(5 \%)$ \\
\hline Delayed gastric emptying $^{d}$ & $25(17 \%)$ \\
\hline Postoperative bleeding ${ }^{\mathrm{e}}$ & $3(2 \%)$ \\
\hline Sepsis $^{f}$ & $33(22 \%)$ \\
\hline Pulmonary edema ${ }^{g}$ & $9(6 \%)$ \\
\hline Myocardial infarction ${ }^{\text {h }}$ & $12(8 \%)$ \\
\hline Cardiac arrythmias ${ }^{i}$ & 19(13) \\
\hline Respiratory failure & $12(8 \%)$ \\
\hline Pulmonary congestion ${ }^{k}$ & $15(10 \%)$ \\
\hline Pneumonial & $26(17 \%)$ \\
\hline Renal failure $^{m}$ & $11(7 \%)$ \\
\hline Urinary tract infection $^{n}$ & $4(3 \%)$ \\
\hline Postoperative delerium ${ }^{\circ}$ & $6(4 \%)$ \\
\hline
\end{tabular}

Patient outcomes

Return to theatre

Unexpected return to intensive care unit

Medical emergency/response call

Death within 30 days

Data presented as number (\%).

anternational Study Group of Pancreatic Fistula (ISGPF).

bresence of bile in the drainage fluid that persisted on postoperative day 4 .

CElevations in serum lipase $>3 \times$ normal laboratory reference range.

${ }^{\mathrm{d} I n t e r n a t i o n a l ~ S t u d y ~ G r o u p ~ o f ~ P a n c r e a t i c ~ F i s t u l a ~(I S G P F) . ~}$

epostoperative blood loss requiring a blood transfusion.

fSurviving sepsis campaign: international guidelines definition.

${ }^{9}$ Radiological features of acute pulmonary edema requiring medical intervention.

${ }^{h} E C G$ changes with myocardial enzyme elevation.

'New onset atrial fibrillation or ventricular arrhythmia requiring medical treatment or cardioversion.

${ }^{\mathrm{j}}$ Prolonged ventilation or reintubation or $\mathrm{PaO}_{2} \leq 50 \mathrm{mmHg}$ or $\mathrm{PaCO}_{2} \geq$

$50 \mathrm{mmHg}$ (room air).

${ }^{\mathrm{k}}$ Shortness of breath with crepitations and desaturation requiring medical intervention.

'Elevated temperature with radiographic pulmonary changes.

${ }^{m}$ Rise in serum creatinine (absolute increase in serum creatinine of $\geq 0.3 \mathrm{mg} / \mathrm{dl}$

$(\geq 26.4 \mu \mathrm{mol} / \mathrm{l})$ or percentage increase in serum creatinine of $\geq 50 \%$ ) or oliguria (urine output $<0.5 \mathrm{ml} / \mathrm{kg} / \mathrm{h}$ for more than 6 hours).

${ }^{n}$ Positive urine culture for pathogens requiring antibiotics.

Impaired cognition, fluctuating level of consciousness with altered psychomotor activity not related to emergence from anesthesia or an identifiable etiology. 
Surgery: RELIEF Study" protocol [9]. A statistical software package (SPSS Version 19.0; IBM Co, Armonk, NY, USA) was used for statistical analysis, with a two-tailed $\mathrm{P}$ value less than 0.05 as statistically significant. Results were expressed as either a median (range) or in the form of frequencies unless otherwise stated. Comparisons between categorical variables were determined by chi-square and Fisher's exact test as appropriate. Non-categorical variables were assessed by the Mann-Whitney $U$ test. Multivariate analysis was undertaken using a backward stepwise logistic regression model to identify factors associated with postoperative discharge by day 14 , including all factors where the $P$ value was less than 0.1 on univariate analysis. A cut off of 14 days represented the median length of stay for patients undergoing uncomplicated PD at our institution [1]. Odds ratios (OR) and 95\% confidence intervals $(\mathrm{CI})$ were reported where appropriate.

\section{Results}

One hundred and fifty consecutive patients undergoing PD were evaluated between 2006 and 2012. The majority of patients were male (59\%), middle-aged (mean: 66 years old), overweight (mean: BMI $26.1 \mathrm{~kg} / \mathrm{m}^{2}$ ) and ASA class III (76\%) (Table 2). Details of postoperative complications are summarised in Table 1. Postoperative complications were frequent and occurred in 86 patients (57\%). The majority of complications were graded as ClavienDindo Class 2 and 3 (Table 1). Postoperative pancreatic fistula occurred in 13 patients (9\%), and delayed gastric emptying occurred in 25 patients (17\%). Other documented postoperative surgical complications included sepsis $(22 \%)$, bile leak (4\%), postoperative bleeding $(2 \%)$ and acute pancreatitis (5\%). Serious medical complications included pulmonary edema (6\%), myocardial infarction (8\%), respiratory failure (8\%), and renal failure (7\%). Broadly, the characteristics of patients with complications were similar to patients without complications (Table 3).

The operative details and pathologies of patients undergoing PD with and without complications are summarised in Table 4. Surgeons who performed fewer pancreaticoduodenectomies appeared to have higher complication rates than surgeons with higher surgical caseloads $(\mathrm{p}<0.001)$. Patients without complications had a higher median estimated blood loss $(400 \mathrm{ml}$; range $200-2500 \mathrm{ml}$ vs. $350 \mathrm{~mL}$; range $100-1900 \mathrm{ml}, \mathrm{p}=0.027$ ), however blood transfusion requirements were similar in both groups (19\%). Intraoperatively patients with complications were more likely to receive a liberal fluid intervention regime (median 5.4 litres; range 2.5-12.3 litres vs. 5.0 litres; range 1.0-10.6 litres; $\mathrm{p}=0.047$ ), and were in a more positive fluid balance (median 4.7 litres; range 1.6-12.0 litres vs. 4.1 litres; range 0.2-9.4 litres; $\mathrm{p}=$ 0.044) compared to patients without complications. Complication rates were similar in patients who received intrathecal morphine or epidural anesthesia compared to

Table 2 Characteristics of patients undergoing pancreaticoduodenal resection with and without complications

\begin{tabular}{|c|c|c|c|c|}
\hline & $\begin{array}{l}\text { Overall } \\
(n=150)\end{array}$ & $\begin{array}{l}\text { Complications } \\
\qquad(n=86)\end{array}$ & $\begin{array}{l}\text { No complications } \\
\qquad(n=64)\end{array}$ & $p$ value \\
\hline \multicolumn{5}{|c|}{ Patient characteristics } \\
\hline Male & 89 (59\%) & $53(62 \%)$ & $36(56 \%)$ & 0.507 \\
\hline Age & $67(15-84)$ & $67(41-84)$ & $66(15-82)$ & 0.496 \\
\hline BMI & $26(18-42)$ & $26(18-37)$ & $24(19-42)$ & $0.046^{*}$ \\
\hline ASA Class I & $1(1 \%)$ & $1(1 \%)$ & $0(0 \%)$ & 1.0 \\
\hline$\|$ & $35(23 \%)$ & $20(23 \%)$ & $15(23 \%)$ & \\
\hline III & $114(76 \%)$ & $65(76 \%)$ & $49(77 \%)$ & \\
\hline Diabetes & $35(23 \%)$ & $24(28 \%)$ & $11(17 \%)$ & 0.125 \\
\hline COPD & $12(8 \%)$ & $9(11 \%)$ & $3(5 \%)$ & 0.237 \\
\hline \multicolumn{5}{|c|}{ Pre-operative laboratory tests } \\
\hline Hemoglobin (g/l) & $130(79-173)$ & $130(82-173)$ & $129(79-156)$ & 0.410 \\
\hline WCC $\left(\times 10^{9} / \mathrm{l}\right)$ & $7.5(3.0-31.6)$ & $7.6(4.1-31.6)$ & $7.0(3.0-16.8)$ & 0.160 \\
\hline Platelets $\left(\times 10^{9} / 1\right)$ & $292(21-744)$ & $293(21-733)$ & $290(139-744)$ & 0.605 \\
\hline Bilirubin ( $\mu \mathrm{mol} / \mathrm{l})$ & $29(5-405)$ & $35(5-405)$ & $19(6-352)$ & 0.109 \\
\hline Albumin (g/l) & $34(13-49)$ & $34(20-46)$ & $35(13-49)$ & $0.045^{*}$ \\
\hline Urea $(\mathrm{mmol} / \mathrm{l})$ & $5.3(0.9-15.4)$ & $5.3(0.9-12.2)$ & $5.2(1.1-15.4)$ & 0.987 \\
\hline Creatinine $(\mu \mathrm{mol} / \mathrm{l})$ & $76(10-241)$ & $79(10-241)$ & $70(28-186)$ & $0.036^{*}$ \\
\hline
\end{tabular}

ASA - American society of anesthesiologists; $B M I$ - body mass index; $W C C$ - white cell count, COPD - Chronic obstructive pulmonary disease; Missing values; $B M I-24$ Hemoglobin 5 WCC 9 Platelets 7 Bilirubin 32, Albumin 35, Urea 10, Creatinine $10 * \mathrm{p} \leq 0.05$ Chi-Square/Fisher's exact test/Mann-Whitney $U$ test. 
Table 3 Characteristics of patients undergoing pancreaticoduodenal resection with and without complications

\begin{tabular}{|c|c|c|c|c|}
\hline & $\begin{array}{l}\text { Overall } \\
(n=150)\end{array}$ & $\begin{array}{l}\text { Complications } \\
\qquad(\mathrm{n}=86)\end{array}$ & $\begin{array}{l}\text { No complications } \\
\qquad(n=64)\end{array}$ & $\mathrm{p}$ value \\
\hline \multicolumn{5}{|c|}{ Patient characteristics } \\
\hline Male & 89 (59\%) & $53(62 \%)$ & $36(56 \%)$ & 0.507 \\
\hline Age & $67(15-84)$ & $67(41-84)$ & $66(15-82)$ & 0.496 \\
\hline BMl & $26(18-42)$ & $26(18-37)$ & $24(19-42)$ & $0.046^{*}$ \\
\hline ASA Class I & $1(1 \%)$ & $1(1 \%)$ & $0(0 \%)$ & 1.0 \\
\hline$\|$ & $35(23 \%)$ & $20(23 \%)$ & $15(23 \%)$ & \\
\hline III & $114(76 \%)$ & $65(76 \%)$ & $49(77 \%)$ & \\
\hline Diabetes & $35(23 \%)$ & $24(28 \%)$ & $11(17 \%)$ & 0.125 \\
\hline COPD & $12(8 \%)$ & $9(11 \%)$ & $3(5 \%)$ & 0.237 \\
\hline \multicolumn{5}{|c|}{ Preoperative laboratory tests } \\
\hline Hemoglobin (g/l) & $130(79-173)$ & $130(82-173)$ & $129(79-156)$ & 0.410 \\
\hline WCC $\left(\times 10^{9} / l\right)$ & $7.5(3.0-31.6)$ & $7.6(4.1-31.6)$ & $7.0(3.0-16.8)$ & 0.160 \\
\hline Platelets $\left(\times 10^{9} / 1\right)$ & $292(21-744)$ & $293(21-733)$ & $290(139-744)$ & 0.605 \\
\hline Bilirubin $(\mu \mathrm{mol} / \mathrm{l})$ & $29(5-405)$ & $35(5-405)$ & $19(6-352)$ & 0.109 \\
\hline Albumin (g/l) & $34(13-49)$ & $34(20-46)$ & $35(13-49)$ & $0.045^{*}$ \\
\hline Urea $(\mathrm{mmol} / \mathrm{l})$ & $5.3(0.9-15.4)$ & $5.3(0.9-12.2)$ & $5.2(1.1-15.4)$ & 0.987 \\
\hline Creatinine $(\mu \mathrm{mol} / \mathrm{l})$ & $76(10-241)$ & $79(10-241)$ & $70(28-186)$ & $0.036^{*}$ \\
\hline
\end{tabular}

those who did not. There was no difference in the use of intraoperative inotropes or vasoconstrictors for patients with or without complications (Table 4).

Table 5 summarizes the detailed administration of postoperative intravenous fluids in the first three postoperative days in patients with and without complications. The majority of fluids given were in the form of crystalloids. The overall median volumes of intravenous fluids given on the first three postoperative days were 3.0 litres on day 1 (range 0.9-14.1 litres), 2.1 litres on day 2 (range 0.3-6.1 litres), and 1.7 litres on day 3 (range 0-6.0 litres). On all three postoperative days, patients with complications received a higher median volume of intravenous therapy (day 1: 3.3 litres vs. 2.9 litres, $\mathrm{p}=0.020$; day 2: 2.3 litres vs.1.9 litres, $\mathrm{p}=0.026$; day 3: 1.9 litres vs.1.4 litres, $\mathrm{p}=0.018$ ) and had higher cumulative positive fluid balances when compared to patients without complications (Table 5). Of interest, within the complication group itself, the fluid balance in patients with ClavienDindo grade 1 and 2 complications were not significantly different to those with grade 3,4 or 5 complications. Postoperative length of stay was significantly greater in patients with complications when compared to patients without complications (median 25 days vs. 10 days; $\mathrm{p}<0.001$ ). Factors associated with complications were creatinine $>100 \mu \mathrm{mmol} / \mathrm{L}$, liberal fluid intervention, positive cumulative fluid balance, and a low individual surgical caseloads (Table 6). After adjustment for covariates, a fluid balance of less than 1 litre on postoperative day 1 , and low surgeon caseloads remained strongly associated with the development of complications. Similarly, a fluid balance of less than 1 litre on postoperative day 1 (OR 2.9; 95\% CI: 1.1-6.6, $\mathrm{p}=0.037$ ), absence of complications (OR $0.1 ; 95 \%$ CI $0.0-0.2 ; \mathrm{p}<0.001)$ and high surgeon caseloads (OR 9.8; 95\% CI 3.3-33.8; $\mathrm{p}<0.001$ ) remained strongly associated with an earlier hospital discharge (Table 7). There were no significant differences in any of the outcomes reported when factoring in time effects over the 7-year study period.

\section{Discussion}

We performed a retrospective study of detailed fluid intervention, complications and length of hospital stay in patients undergoing PD. We found that, as hypothesized, restrictive fluid intervention and a neutral/negative cumulative fluid balance were associated with reduced complications and shorter length of hospital stay. Importantly, we found that improvement in PD outcomes occurred with increased surgical caseload, and surgeon experience remained an important determinant of overall morbidity.

The demographic and clinical features of our patients are consistent with other studies of similar types of surgery $[1,10-13]$. Likewise our complication rates appear to be similar to other university hospital hepatobiliary units $[14,15]$. However, as there are no studies assessing the 
Table 4 Operative and pathology of patients undergoing pancreaticoduodenal resection with and without complications

\begin{tabular}{|c|c|c|c|c|}
\hline & $\begin{array}{l}\text { Overall } \\
(n=150)\end{array}$ & $\begin{array}{l}\text { Complications } \\
\qquad(\mathrm{n}=86)\end{array}$ & $\begin{array}{l}\text { No complications } \\
\qquad(n=64)\end{array}$ & $p$ value \\
\hline Malignancy & $125(83 \%)$ & $74(86 \%)$ & $51(80 \%)$ & 0.301 \\
\hline Epidural anesthesia & $84(57 \%)$ & $45(53 \%)$ & $39(62 \%)$ & 0.276 \\
\hline Intrathecal morphine & $20(13 \%)$ & $8(9 \%)$ & $12(19 \%)$ & 0.092 \\
\hline Pylorus preserving & $63(42 \%)$ & $40(47 \%)$ & $23(36 \%)$ & 0.194 \\
\hline Surgeons with higher surgical volumes* & & & & $0.001^{*}$ \\
\hline Surgeon 1 & $52(35 \%)$ & $17(20 \%)$ & $35(55 \%)$ & \\
\hline Surgeon 2 & $29(19 \%)$ & 19 (22\%) & $10(16 \%)$ & \\
\hline Surgeon 3 & $13(9 \%)$ & $8(9 \%)$ & $5(8 \%)$ & \\
\hline Surgeon 4 & $9(6 \%)$ & $6(7 \%)$ & $3(5 \%)$ & \\
\hline Surgeon 5 & $20(13 \%)$ & $15(17 \%)$ & $5(8 \%)$ & \\
\hline Other & $27(18 \%)$ & $21(24 \%)$ & $6(9 \%)$ & \\
\hline Estimated blood loss (ml) & $350(100-2500)$ & $350(100-1900)$ & $400(200-2500)$ & $0.027^{*}$ \\
\hline Blood transfusions intraoperative & $28(19 \%)$ & $16(19 \%)$ & $12(19 \%)$ & 0.982 \\
\hline Intraoperative fluids (I) & $5.0(1.0-12.3)$ & $5.4(2.5-12.3)$ & $5.0(1.0-10.6)$ & $0.047^{*}$ \\
\hline Fluid balance (I) & $4.5(0.2-12.0)$ & $4.7(1.6-12.0)$ & $4.1(0.2-9.4)$ & $0.044^{*}$ \\
\hline \multicolumn{5}{|l|}{ Inotropes and vasoconstrictors } \\
\hline Total use & $90(60 \%)$ & $55(64 \%)$ & $35(55 \%)$ & \multirow{5}{*}{0.89} \\
\hline Norepinephrine & $8(5 \%)$ & $5(6 \%)$ & $3(5 \%)$ & \\
\hline Metaraminol & $70(47 \%)$ & $39(45 \%)$ & $31(48 \%)$ & \\
\hline Ephedrine & $15(10 \%)$ & $9(10 \%)$ & $8(13 \%)$ & \\
\hline Dopamine & $5(3 \%)$ & $3(3 \%)$ & $2(3 \%)$ & \\
\hline Operative time (hours) & $7.0(3-15.8)$ & $6.6(3-15.8)$ & $7.2(3-12)$ & 0.056 \\
\hline
\end{tabular}

*Defined as greater than 10 pancreatic resections per annum.

effects of perioperative cumulative fluid balances on adverse outcomes and length of hospital stay following PD, direct comparisons are not possible. However, a recent study by Melis et al. examined the influence of intraoperative crystalloid administration on complications following PD for pancreatic adenocarcinoma [11]. The volume of intraoperative crystalloid administered increased with duration of surgery, intraoperative blood loss and intra-operative blood transfusion, but unlike our data, this did not correlate with postoperative morbidity. However, perioperative fluid balances were not reported. Perioperative cumulative fluid balance has been shown to be an important predictor of surgical outcomes and can be used as a prognostic tool to evaluate the risk of surgical complications [12]. Our study supports these findings that a positive cumulative fluid balance is associated with more complications and a longer length of hospital stay. We found the difference in liberal intravenous fluid intervention to be most apparent in the postoperative setting. Even after adjustment for covariates, a positive fluid balance on postoperative day 1 remained strongly associated with of the length of hospital stay. Patients with postoperative complications had a longer length of hospital stay compared to patients without complications (median 25 days vs. 10 days; $\mathrm{p}=$ 0.001). This finding is similar to data from other multicentre Australian studies [13]. Importantly, complication rates of $\mathrm{PD}$ and the associated length of hospital stay continue to affect patient outcomes and strain limited healthcare resources [14].

Similar to other studies we found the surgical case load to have a significant impact on perioperative morbidity following PD [16-19]. Pancreaticoduodenectomy has an inherent learning curve and it has been suggested that after sixty cases, surgeons performing PD achieve significantly decreased blood loss, operative time, and length of hospital stay, and carry out more marginnegative resections [16-19].

There are several limitations of our study. Although this is the largest study examining the association of detailed fluid intervention, fluid balance and surgical outcomes in patients undergoing PD, only 150 patient records were reviewed. Data was collected from a hospital maintained database, which limited our ability to recover any missing or unclear data. Similar to a previous study [1], it is possible that not all complications 
Table 5 Postoperative and outcome details of patients undergoing pancreaticoduodenal resection with and without complications

\begin{tabular}{|c|c|c|c|c|}
\hline & $\begin{array}{l}\text { Overall } \\
(n=150)\end{array}$ & $\begin{array}{l}\text { Complications } \\
\qquad(n=86)\end{array}$ & $\begin{array}{l}\text { No complications } \\
\qquad(n=64)\end{array}$ & $p$ value \\
\hline Day 1 total fluids (litres) & $3.0(0.9-14.1)$ & $3.3(0.9-14.1)$ & $2.9(1.4-11.0)$ & $0.020^{*}$ \\
\hline - Crystalloid & $2.7(0.9-13.1)$ & $2.8(0.9-13.1)$ & $2.6(1.3-10.0)$ & 0.101 \\
\hline - Colloid & $0.0(0.0-2.8)$ & $0.0(0.0-2.8)$ & $0.0(0.0-2.0)$ & 0.952 \\
\hline - Blood & $0.0(0.0-1.1)$ & $0.0(0.0-1.1)$ & $0.0(0.0-0.3)$ & 0.239 \\
\hline - Fluid balance & $1.5(-1.7-12.1)$ & $1.8(-1.7-12.1$ & $0.9(-0.8-8.4)$ & $0.002^{*}$ \\
\hline Day 2 total fluids (litres) & $2.1(0.3-6.1)$ & $2.3(0.3-6.1)$ & $1.9(0.7-4.9)$ & $0.026^{*}$ \\
\hline - Crystalloid & $2.0(0.3-5.5)$ & $2.2(0.4-5.5)$ & $1.90 .7-4.4)$ & $0.009^{*}$ \\
\hline - Colloid & $0.0(0.0-1.9)$ & $0.0(0.0-1.9)$ & $0.0(0.0-0.7)$ & 0.146 \\
\hline - Blood & $0.0(0.0-0.6)$ & $0.0(0.0-0.6)$ & $0.0(0.0-0.5)$ & 0.866 \\
\hline - Fluid balance & $0.4(-2.9-4.5)$ & $0.6(-2.9-4.5)$ & $0.2(-2.4-5.3)$ & $0.037^{*}$ \\
\hline Day 3 total fluids (litres) & $1.7(0.0-6.0)$ & $1.9(0.1-6.0)$ & $1.4(0.0-4.0)$ & $0.018^{*}$ \\
\hline - Crystalloid & $1.6(0.0-6.0)$ & $1.9(0.1-6.0)$ & $1.4(0-4.0)$ & $0.028^{*}$ \\
\hline - Colloid & $0.0(0.0-1.3)$ & $0.0(0.0-1.1)$ & $0.0(0.0-1.3)$ & 0.220 \\
\hline - Blood & $0.0(0.0-0.8)$ & $0.0(0.0-0.8)$ & $0.0(0.0 .4)$ & 0.727 \\
\hline - Fluid balance & $0.4(-2.9-11.9)$ & $0.5(-2.6-11.9)$ & $0.2(-2.9-11.7)$ & 0.103 \\
\hline Postoperative length of stay (days) & $17(6-140)$ & $25(7-140)$ & $10(6-90)$ & $<0.001$ \\
\hline
\end{tabular}

were properly recorded. The pancreatic leak rate in particular appears to identify only patients with Grade B, C leaks, with the possibility that Grade A leaks have been under-reported. However, this would simply reinforce the contention that these patients experience a high level of postoperative complications. There may also have been inaccuracies in the recording of fluid therapy. We consider this unlikely due to our comprehensive intraoperative fluid therapy documentation practices, cross checks, and computerized medical records. In addition, the medical records were reviewed by three independent investigators who checked that a thorough and accurate tally of fluid administration and losses was recorded. This is a single centre study, which may limit the

Table 6 Factors associated with overall complications following pancreaticoduodenectomy

\begin{tabular}{|c|c|c|c|c|c|c|}
\hline & \multirow{2}{*}{$\begin{array}{l}\text { Complications } \\
\qquad(n=86)\end{array}$} & \multirow{2}{*}{$\begin{array}{l}\text { No complications } \\
\qquad(n=64)\end{array}$} & \multicolumn{2}{|l|}{ Univariate } & \multicolumn{2}{|l|}{ Multivariate } \\
\hline & & & $\begin{array}{c}\text { Odds ratio } \\
\text { (Confidence interval) }\end{array}$ & $p$ value & $\begin{array}{c}\text { Odds ratio } \\
\text { (Confidence interval) }\end{array}$ & $p$ value \\
\hline \multicolumn{7}{|l|}{ Demographics } \\
\hline $\mathrm{BMI} \geq 25$ & $43(62 \%)$ & $28(49 \%)$ & $1.7(0.8-3.5)$ & 0.137 & & \\
\hline Albumin $\leq 30 \mathrm{~g} / \mathrm{l}$ & $19(34 \%)$ & $9(15 \%)$ & $2.9(1.2-7.40$ & $0.020^{*}$ & $1.6(0.6-4.6)$ & 0.389 \\
\hline Creatinine $>100(\mu \mathrm{mol} / \mathrm{l})$ & $17(22 \%)$ & $6(10 \%)$ & $2.6(1.0-7.1)$ & 0.055 & $3.9(1.1-13.4)$ & $0.033^{*}$ \\
\hline \multicolumn{7}{|l|}{ Operative details } \\
\hline Time $\geq 8$ hours & $24(28 \%)$ & $25(39 \%)$ & $0.6(0.3-1.2)$ & 0.150 & & \\
\hline Blood loss $\geq 600 \mathrm{ml}$ & $15(18 \%)$ & $14(22 \%)$ & $0.8(0.4-1.7)$ & 0.510 & & \\
\hline Intraoperative fluid balance $\leq 3$ litre & $29(34 \%)$ & $32(50 \%)$ & $0.3(0.1-0.7)$ & $0.006^{*}$ & $0.6(0.2-1.7)$ & 0.367 \\
\hline High surgical volume & $12(14 \%)$ & $21(33 \%)$ & $0.2(0.1-0.4)$ & $<0.001^{*}$ & $0.2(0.1-0.5)$ & $0.001^{*}$ \\
\hline \multicolumn{7}{|l|}{ Post-operative details } \\
\hline Day 1 fluid balance $\leq 1$ litre & $26(30 \%)$ & $36(56 \%)$ & $0.4(0.2-0.7)$ & $0.001^{*}$ & $0.2(0.1-0.6)$ & $0.001 *$ \\
\hline Day 2 fluid balance $\leq 0.2$ litre & $28(33 \%)$ & $32(50 \%)$ & $0.5(0.3-0.9)$ & $0.031^{*}$ & $0.8(0.3-2.1)$ & 0.685 \\
\hline Day 3 fluid balance $\leq 0.2$ litre & $36(42 \%)$ & $32(50 \%)$ & $0.7(0.4-1.4)$ & 0.32 & & \\
\hline
\end{tabular}

Data are presented as number (\%) or median (range).

$B M I$ - body mass index; Missing: BMI - 24, Albumin -35, Creatinine 10, Estimate blood loss - 3 * $\mathrm{p} \leq 0.05$; Chi-Square/Fisher's exact test. 
Table 7 Factors associated with post-operative length of two weeks or less following pancreaticoduodenectomy

\begin{tabular}{|c|c|c|c|c|c|c|}
\hline & \multirow[b]{2}{*}{$\begin{array}{l}\text { Discharge before } \\
2 \text { weeks }\end{array}$} & \multirow{2}{*}{$\begin{array}{l}\text { Discharge after } \\
2 \text { weeks }\end{array}$} & \multicolumn{2}{|l|}{ Univariate } & \multicolumn{2}{|l|}{ Multivariate } \\
\hline & & & $\begin{array}{c}\text { Odds ratio } \\
\text { (Confidence interval) }\end{array}$ & $p$ value & $\begin{array}{c}\text { Odds ratio } \\
\text { (Confidence interval) }\end{array}$ & $p$ value \\
\hline \multicolumn{7}{|l|}{ Demographics } \\
\hline Male & $41(66 \%)$ & $48(55 \%)$ & $1.6(0.8-3.2)$ & 0.155 & & \\
\hline Diabetes & $16(26 \%)$ & $19(22 \%)$ & $1.3(0.6-2.7)$ & 0.548 & & \\
\hline COPD & $2(3 \%)$ & $10(11 \%)$ & $0.3(0.1-1.2)$ & 0.124 & & \\
\hline Age $\geq 70$ & $20(32 \%)$ & $38(43 \%)$ & $0.6(0.3-1.2)$ & 0.176 & & \\
\hline $\mathrm{BMI} \geq 25$ & $29(50 \%)$ & $42(62 \%)$ & $0.6(0.3-1.3)$ & 0.184 & & \\
\hline Albumin $\leq 30 \mathrm{~g} / \mathrm{l}$ & $12(21 \%)$ & $16(28 \%)$ & $0.7(0.3-1.7)$ & 0.414 & & \\
\hline Creatinine $\geq 100(\mu \mathrm{mol} / \mathrm{l})$ & $6(10 \%)$ & $17(21 \%)$ & $0.4(0.2-1.2)$ & 0.088 & $0.3(0.1-1.2)$ & 0.085 \\
\hline \multicolumn{7}{|l|}{ Operative details } \\
\hline Time $\geq 8$ hours & $24(39 \%)$ & $25(28 \%)$ & $1.6(0.8-3.1)$ & 0.185 & & \\
\hline Blood loss $\geq 600 \mathrm{ml}$ & $9(15 \%)$ & $20(23 \%)$ & $0.6(0.2-1.4)$ & 0.202 & & \\
\hline Intraoperative fluid balance $\leq 3$ litre & $20(32 \%)$ & $13(15 \%)$ & $2.7(1.2-6.1)$ & $0.01^{*}$ & $1.7(0.5-5.1)$ & 0.382 \\
\hline High surgical volume & $39(63 \%)$ & $13(25 \%)$ & $9.8(4.5-21.4)$ & $<0.001^{*}$ & $11.3(3.8-33.8)$. & $<0.001^{*}$ \\
\hline Malignancy & 49 (79\%) & $76(86 \%)$ & $0.6(0.3-1.4)$ & 0.235 & & \\
\hline \multicolumn{7}{|l|}{ Post-operative details } \\
\hline Complication & $15(24 \%)$ & $71(80 \%)$ & $0.1(0.0-0.2)$ & $<0.001^{*}$ & $0.1(0.1-0.4)$ & $<0.001^{*}$ \\
\hline Day 1 fluid balance $\leq 1$ litre & $35(57 \%)$ & $27(31 \%)$ & $2.9(1.5-5.8)$ & $0.002^{*}$ & $2.9(1.1-6.6)$ & $0.037^{*}$ \\
\hline Day 2 fluid balance $\leq 0.2$ litre & $33(53 \%)$ & $37(31 \%)$ & $2.6(1.3-5.0)$ & $0.006^{*}$ & $0.8(0.3-2.5)$ & 0.752 \\
\hline Day 3 fluid balance $\leq 0.2$ litre & $36(58 \%)$ & $32(36 \%)$ & $2.4(1.2-4.7)$ & $0.009^{*}$ & $2.5(1.0-6.6)$ & 0.056 \\
\hline
\end{tabular}

external validity of our findings. However, our hospital has all the typical characteristics of a tertiary institution in a developed country and a recent comparative study confirmed that its patients and their outcomes were equivalent to those of other tertiary hospitals in Australia [20]. Our study has several strengths. Information on detailed perioperative fluid intervention and fluid balances provide a background for power calculations needed to design future prospective fluid interventional trials in this group of patients. Finally, by defining the complication and mortality rate in these patients, we have identified a need for improved perioperative care, and a possible pathway to achieve this goal.

Whilst the findings of this study suggest that positive fluid balance is associated with postoperative complications and increased length of hospital stay in the setting of PD, this does not imply causality. Increased perioperative fluid intervention may occur as a consequence of complications. Sepsis, peritonitis, renal and cardiac failure, and pancreatic leak all can cause fluid retention and/or edema. Fluid balance may therefore simply be a marker of illness rather than the cause. It is also plausible that both of these mechanisms co-exist. Whilst there is emerging evidence that early goal directed therapy and fast track programs improve surgical outcomes and reduce postoperative hospital stay [21,22], there is limited information on detailed fluid intervention and cumulative fluid balance in the context of PD.

\section{Conclusions}

Therefore, these results demonstrate that a high surgical caseload and a restrictive perioperative fluid intervention regime, with negative cumulative fluid balance were associated with fewer complications and shorter length of hospital stay. These findings, specific to patients undergoing PD, are of particular interest as the higher rates of complications and prolonged length of stay provide good opportunities to evaluate strategies aimed at improving perioperative care for this group.

\section{Competing interests}

The authors declare that they have no competing interests.

\section{Authors' contributions}

LW and MN conceived the study, participated in the final planning and study design, performed data collection and all statistical analyses; they were responsible for the final writing of the manuscript. DW, BP, COT, ST were responsible for the collection of anesthesia data, cross checking of all 
anesthesia records with medical records, and calculation and checking of all fluid balances. They also assisted with data entry into a database and the writing of the manuscript. DK and RB were the responsible for the collection of data in the intensive care and high dependency units, entering of data into the master database, interpretation of data, and assistance with the writing of the manuscript. CC, LM and MN were responsible for the collection of all surgical outcomes and lengths of hospital stay. They cross checked surgical records with hospital medical records. In addition they assisted with the writing of the manuscript. All authors read and approved the final manuscript.

\section{Author details}

'Department of Anesthesia, Department of Surgery, University of Melbourne, Austin Hospital, Heidelberg, Australia. ${ }^{2}$ Department of Anesthesia, Austin Hospital, Heidelberg, Australia. ${ }^{3}$ Department of Anesthesia \& Intensive Care Medicine, Austin Hospital, Heidelberg, Australia. ${ }^{4}$ Department of Anesthesia, Austin Hospital, Melbourne, Australia. ${ }^{5}$ Department of Anesthesia, Royal Darwin Hospital, Darwin, Australia. ${ }^{6}$ University of Melbourne, Austin Hospital, Heidelberg, Australia. ${ }^{7}$ Department of Surgery, University of Melbourne, Heidelberg, Australia.

Received: 31 August 2013 Accepted: 7 May 2014

Published: 14 May 2014

\section{References}

1. Warrillow SJ, Weinberg L, Parker F, Calzavacca P, Licari E, Aly A, Bagshaw S, Christophi C, Bellomo R: Perioperative fluid prescription, complications and outcomes in major elective open gastrointestinal surgery. Anaesth Intensive Care 2010, 38:259-265.

2. Nikfarjam M, Weinberg L, Low N, Fink MA, Muralidharan V, Houli N, Starkey G, Jones R, Christophi C: A fast track recovery program significantly reduces hospital length of stay following uncomplicated pancreaticoduodenectomy. JOP 2013, 14:63-70.

3. Berberat PO, Ingold H, Gulbinas A, Kleeff J, Müller MW, Gutt C, Weigand M, Friess H, Büchler MW: Fast track-different implications in pancreatic surgery. J Gastrointest Surg 2007, 11:880-887.

4. Pratt WB, Maithel SK, Vanounou T, Huang ZS, Callery MP, Vollmer CM Jr: Clinical and economic validation of the International Study Group of Pancreatic Fistula (ISGPF) classification scheme. Ann Surg 2007, 245:443-451.

5. Wente MN, Bassi C, Dervenis C, Fingerhut A, Gouma DJ, Izbicki JR, Neoptolemos JP, Padbury RT, Sarr MG, Traverso LW, Yeo CJ, Büchler MW: Delayed gastric emptying (DGE) after pancreatic surgery: a suggested definition by the International Study Group of Pancreatic Surgery (ISGPS). Surgery 2007, 142:761-768.

6. Tan WJ, Kow AW, Liau KH: Moving towards the New International Study Group for Pancreatic Surgery (ISGPS) definitions in pancreaticoduodenectomy: a comparison between the old and new. HPB (Oxford) 2011, 13:566-572.

7. Dindo D, Demartines N, Clavien PA: Classification of surgical complications: a new proposal with evaluation in a cohort of 6336 patients and results of a survey. Ann Surg 2004, 240:205-213.

8. Common Terminology Criteria for Adverse Events (CTCAE), Version 4. US Department of Health and Human Services, National Institute of Health and National Cancer Institute; Accessed 10 March 2014 http://www.acrin.org/ Portals/O/Administration/Regulatory/CTCAE_4.02_2009-09-15_QuickReference_ 5x7.pdf.

9. Myles PS: REstrictive Versus LIbEral Fluid Therapy in Major Abdominal Surgery: RELIEF Study. 2013, http://clinicaltrials.gov/show/NCT01424150 (accessed 16/6/2013).

10. Brandstrup B, Tønnesen H, Beier-Holgersen R, Hjortsø E, Ørding H, LindorffLarsen K, Rasmussen MS, Lanng C, Wallin L, Iversen LH, Gramkow CS, Okholm M, Blemmer T, Svendsen PE, Rottensten HH, Thage B, Riis J, Jeppesen IS, Teilum D, Christensen AM, Graungaard B, Pott F, Danish Study Group on Perioperative Fluid Therapy: Effects of intravenous fluid restriction on postoperative complications: comparison of two perioperative fluid regimens: a randomised assessor-blinded multicentre trial. Ann Surg 2003, 238:641-648.

11. Melis M, Marcon F, Masi A, Sarpel U, Miller G, Moore H, Cohen S, Berman R, Pachter $\mathrm{HL}$, Newman E: Effect of intraoperative fluid volume on perioperative outcomes after pancreaticoduodenectomy for pancreatic adenocarcinoma. J Surg Oncol 2012, 105:81-84.

12. Wei $S$, Tian J, Song $X$, Chen Y: Association of perioperative fluid balance and adverse surgical outcomes in esophageal cancer and esophagogastric junction cancer. Ann Thorac Surg 2008, 86:266-272.

13. Story DA, Leslie K, Myles PS, Fink M, Poustie SJ, Forbes A, Yap S, Beavis V Kerridge R, REASON Investigators, Australian and New Zealand College of Anaesthetists Trials Group: Complications and mortality in older surgical patients in Australia and New Zealand (the REASON study): a multicentre, prospective, observational study. Anaesthesia 2010, 65:1022-1030.

14. Cameron JL, Riall TS, Coleman J, Belcher KA: One thousand consecutive pancreaticoduodenectomies. Ann Surg 2006, 244:10-15.

15. Bassi C, Falconi M, Salvia R, Mascetta G, Molinari E, Pederzoli P: Management of complications after pancreaticoduodenectomy in a high volume centre: results on 150 consecutive patients/with invited commentary. Dig Surg 2001, 18:453-458.

16. Schmidt CM, Turrini O, Parikh P, House MG, Zyromski NJ, Nakeeb A, Howard TJ, Pitt HA, Lillemoe KD: Effect of hospital volume, surgeon experience, and surgeon volume on patient outcomes after pancreaticoduodenectomy: a single-institution experience. Arch Surg 2010, 145:634-640.

17. Tseng JF, Pisters PW, Lee JE, Wang H, Gomez HF, Sun CC, Evans DB: The learning curve in pancreatic surgery. Surgery 2007, 141:694-701.

18. Rosemurgy A, Cowgill S, Coe B, Thomas A, Al-Saadi S, Goldin S, Zervos E: Frequency with which surgeons undertake pancreaticoduodenectomy continues to determine length of stay, hospital charges, and in-hospital mortality. J Gastrointest Surg 2008, 12:442-449.

19. Joseph B, Morton JM, Hernandez-Boussard T, Rubinfeld I, Faraj C, Velanovich $\checkmark$ : Relationship between hospital volume, system clinical resources, and mortality in pancreatic resection. J Am Coll Surg 2009, 208:520-527.

20. McNicol L, Story DA, Leslie K, Myles PS, Fink M, Shelton AC, Clavisi O, Poustie SJ: Postoperative complications and mortality in older patients having non-cardiac surgery at three Melbourne teaching hospitals. Med J Aust 2007, 186:47-52.

21. Pearse R, Dawson D, Fawcett J, Rhodes A, Grounds RM, Bennett ED: Early goal-directed therapy after major surgery reduces complications and duration of hospital stay. A randomised, controlled trial [ISRCTN38797445]. Crit Care 2005, 9:R687-R693.

22. Weinberg L, Houli N, Nikfarjam M: Improving outcomes for pancreatic cancer: radical surgery with patient-tailored, surgery-specific advanced haemodynamic monitoring. BMJ Case Rep 2013, doi:bcr2013008910.

doi:10.1186/1471-2253-14-35

Cite this article as: Weinberg et al:: The impact of fluid intervention on complications and length of hospital stay after pancreaticoduodenectomy (Whipple's procedure). BMC Anesthesiology 2014 14:35.

\section{Submit your next manuscript to BioMed Central and take full advantage of:}

- Convenient online submission

- Thorough peer review

- No space constraints or color figure charges

- Immediate publication on acceptance

- Inclusion in PubMed, CAS, Scopus and Google Scholar

- Research which is freely available for redistribution 